\title{
Volumenabhängigkeit der Atemmechanik Parameter Resistance und Compliance und ihre Bestimmung bei mechanischer Beatmung mit der SLICE Methode.
}

\author{
J. Guttmann', L. Eberhard², A. Benzing1, G. Mols', M. Lichtwarck-Aschoff ${ }^{3}$, K. Geiger $^{1}$, G. Wolff $^{2}$ \\ 1 Anaesthesiologische Universitätsklinik; Sektion für Experimentelle Anaesthesiologie, \\ Klinikum der Albert-Ludwigs-Universität Freiburg, D-79106 Freiburg i.Br. \\ 2 Klinische Physiologie, Klinik für Herz- und Thoraxchirurgie, Departement Chirurgie, \\ Universitätskliniken Basel, $\mathrm{CH}-4031$ Basel, Schweiz \\ 3 Institut für Anästhesiologie und operative Intensivmedizin, Zentralklinikum Augsburg, \\ D-86156 Augsburg
}

\section{Einleitung}

Die Impedanz des respiratorischen Systems (Lunge und Thorax) besteht aus einem elastischen Teil, einem nicht-elastischen Teil und einem trägheitsbedingten (inertiven) Teil. Letzterer ist bei der mechanischen Beatmung des Erwachsenen zu vernachlässigen (Atemfrequenz $<25 / \mathrm{min}$ ). Der elastische Teil der Impedanz wird als Volumendehnbarkeit oder Compliance (C), der nichtelastische Teil als Strömungswiderstand oder Resistance $(R)$ bezeichnet. Die zugrundeliegende Modellvorstellung ist die des klassischen linearen RCModells, das auf der Voraussetzung beruht, daß R und $C$ während des gesamten Atemzuges konstant sind. Diese Voraussetzung ist bei Patienten mit geringgradiger Lungenerkrankung erfült, nicht jedoch bei Patienten mit schwerer respiratorischer Insuffizienz, wie etwa dem ARDS (adult respiratory distress syndrome). Bei dem stark eingeschränkten Lungengasvolumen im ARDS hängen die mechanischen Eigenschaften des respiratorischen Systems vom Grad seiner mechanischen Auslenkung, d.h. vom Gasvolumen der Lunge ab.

Wir präsentieren eine neue Computer-unterstützte Vielpunkt Methode zur simultanen Bestimmung der volumenabhängigen, dynamischen Resistance und Compliance, die SLICE Methode. Wir präsentieren die Ergebnisse der Methodenevaluierung mit einem physikalischen Modell mit konstanter Resistance und volumenabhăngiger Compliance und mit Messdaten von 14 ARDS Patienten.

\section{Methode}

Die SLICE Methode Ist ein mehrstufiges Verfahren

[1]:

STUFE 1: Erzeugen von volumenáquidistanten Druck-und Fluss Datensâtzen: Um eine Gewlchtung von inspiratorischen oder exspiratorischen Daten auszuschliessen, wird die Anzahl der in- und exspiratorischen Datensamples gleichgesetzt (I-E Balance). Die zeitlich äquidistanten Messdaten von Druck und Gasfluss werden durch lineare Interpolation in volumenäquidistante Datensätze umgewandelt. (Volumenschritt: $\Delta V=10 \mathrm{ml}$ ).

STUFE 2: Punktweise Berechnung des

Trachealdruckes (Ptrach): Mit den volumenäquidistanten Druck- und Flussdaten und den Koeffizienten, die den flussabhängigen Druckabfall über dem Endotrachealtubus beschreiben, wird der Trachealdruck kontinuierlich berechnet [2].

STUFE 3: Unterteilung in Volumenslices und Analyse der Atemmechanik: $90 \%$ des Tidalvolumens werden in sechs Volumenslices gleicher Grösse unterteilt. Die ersten und die letzten $5 \%$ des Tidalvolumens werden nicht analysiert (Ventilartefakte und hohe Volumenbeschleunigung). Die Analyse der Atemmechanik wird separat für jede Volumenslice durchgeführt: multiple lineare Regressionsanalyse [3]. Pro Volumenslice wird ein Resistance- und ein Compliancewert (intrinsic PEEP korrigiert) berechnet. STUFE 4: Qualitätstest: In jeder Volumenslice wird die V/Purach-Kurve mit Hilfe der berechneten R-und CWerte rekonstruiert und der gemessenen V/PtrachKurve überlagert. Je weniger sich beide Kurven hinsichtlich des Druckes unterscheiden, desto besser ist die Qualităt der Ergebnisse der SLICE Methode [6].

Pneumatisches System: Die Untersuchungen zur Evaluierung der SLICE Methode wurden mit einem pnoumatischen System bestehend aus Respirator und Atemschlauchen in Verbindung mit dem passiven physikalischen Modell bzw. in Verbindung 
mit dem passiven respiratorischen System (relaxierte Atemmuskulatur) des ARDS Patienten durchgeführt. Zwischen Respirator und physikalischem Modell bzw. Patient wurden Gasfluss (Fleisch Pneumotachograph No.2) und Druck innerhalb des pneumatischen Systems kontinuierlich gemessen. Die analogen Fluss- und Drucksignale wurden in digitale Signale umgewandelt ( 12 bit) und mit einer Frequenz von 60 $\mathrm{Hz}$ für die offline Analyse gespeichert.

Physikalisches Modell: Für die Laboruntersuchung wurde ein einfaches physikalisches Modell mit konstanter Resistance (2.6 mbar*s/l) und volumenabhängiger Compliance entwickelt. Durch Verānderung der Geometrie des Modells kann dessen Compliance volumenabhängig zwischen 67 $\mathrm{ml} / \mathrm{mbar}$ und $21 \mathrm{ml} / \mathrm{mbar}$ variiert werden. Die Resistance und die Compliance des Modells wurde mit den Ergebnissen der SLICE Methode verglichen.

Patientenuntersuchung: In einer klinischen Studie wurden 14 mechanisch beatmete Patienten mit schwerem ARDS (Murray score $>2.5$ ) untersucht. Die Patienten wurden volumenkontrolliert mit konstantem Inspirationsfluss beatmet. Alle Patienten waren tracheal intubiert. Zur Evaluierung der SLICE Methode wird der end-inspiratorische Alveolardruck (Palv.le) benötigt. Die Patienten wurden deshalb mit einer end-inspiratorischen Pause von $1010 \mathrm{~ms} \pm 100$ $\mathrm{ms}(\mathrm{MW} \pm \mathrm{SD})$ Dauer beatmet. Bei jedem Patienten wurde der gemessene end-inspiratorische Atemwegsdruck ( $P$ aw,le) mit dem berechneten endinspiratorischen Alveolardruck (Palv,le) verglichen. Palv.le wurde als Endpunkt einer rekonstruierten V/PKurve bestimmt, deren Steigung den Compliancewerten entspricht, die mit der SLICE Methode ermittelt wurden. Der Zusammenhang zwischen dem gemessenen Paw,le und dem berechneten $\mathrm{Palvile}$ wurde mit der Regressionsanalyse untersucht.

\section{Ergebnisse}

Physikalisches Modell: Die Resistance und Compliance des Modells wurden mit der SLICE Methode auf $\pm 5 \%$ genau bestimmt. Die mittlere quadratische Abweichung zwischen der volumenabhängigen Compliance des Modells und der berechneten Compliance betrug $2 \mathrm{ml} / \mathrm{mbar}$.

Patientenuntersuchung: Bei den 14 ARDS Patienten wurde eine sehr gute Übereinstimmung zwischen dem gemessenen $P_{a w}$ le und dem berechneten Palv,le

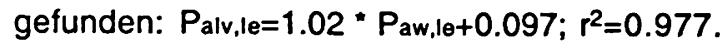

\section{Diskussion}

Computer-unterstützte Vielpunkt Methoden der Atemmechanik Analyse stehen heute am Intensivbett zur Verfügung. Die Methode der multiplen linearen Regression ist eine Vielpunkt Methode, die unabhăngig vom Beatmungsmuster arbeitet und kein zusätzliches Atemmanöver benötigt. Bei der Compliance Bestimmung wird der intrinsic PEEP automatisch berücksichtigt. Ebenso wird der flussabhängige Widerstand des Endotrachealtubus' berücksichtigt. Die Analyse der Atemmechanik wird separat für jede Volumenslice auf der Basis des linearen RC-Modells durchgeführt, d.h. der Gültigkeitsbereich für das lineare Modell wird auf jeweils eine einzelne Volumenslice beschränkt. Die SLICE Methode ermöglicht die Analyse der nichtlinearen. Atemmechanik innerhalb des Tidalvolumens eines individuellen Patienten.

Der relative Fehler der SLICE Methode liegt bei $\pm 5 \%$. Bei den ARDS Patienten haben wir eine sehr gute Übereinstimmung zwischen dem unter statischen Bedingungen $\left(V^{\prime}=0\right)$ gemessenen endinspiratorischen Plateaudruck ( $P$ aw,le) und dem unter dynamischen Bedingungen $\left(V^{\prime} \neq 0\right)$ ermittelten endinspiratorischen Alveolardruck (Palv,le) gefunden.

Die SLICE Methode ermöglicht die manöverfreie, atemzugsweise Analyse der volumenabhängigen Resistance und Compliance und gibt dem Arzt Auskunft darüber, ob der individuelle Patient in dem Bereich seines Lungengasvolumens beatmet wird, in dem die Compliance maximal ist, d.h. im nerwũnschten elastischen Arbeitsbereich".

\section{Literatur}

[1] Guttmann, J.; L. Eberhard, B. Fabry, D. Zappe, H. Bernhard, M. Lichtwarck-Aschoff, M. Adolph, G. Wolff: Determination of volume-dependent respiratory system mechanics in mechanically ventilated patients using the new SLICE method. Technology and Health Care (in press).

[2] Guttmann, J.; L. Eberhard, B. Fabry, W. Bertschmann, G. Wolff: Continuous calculation of intratracheal pressure in tracheally intubated patients. Anesthesiology 79 (1993), 503-513.

[3] Brunner, J. X.; G. Wolff: Pulmonary Function Indices in Critical Care Patients. Springer, Berlin (1988), pp. 18-33. 